\title{
Preparation and characterization of AFM tips with nitrogen- vacancy and nitrogen-vacancy-nitrogen color centers
}

\author{
Zuzanna Orzechowska, ${ }^{1}$ Mariusz Mrózek, ${ }^{1}$ Wojciech Gawlik, ${ }^{1}$ and Adam M. Wojciechowski ${ }^{*}, 1$ \\ ${ }^{I}$ Faculty of Physics, Astronomy and Applied Computer Science, \\ Jagiellonian University, Łojasiewicza 11,30-348 Kraków, Poland
}

Received March 23, 2021; accepted April 28, 2021; published June 30, 2021

\begin{abstract}
We demonstrate a simple dip-coating method of covering standard AFM tips with nanodiamonds containing color centers. Such coating enables convenient visualization of AFM tips above transparent samples as well as tip using for performing spatially resolved magnetometry.
\end{abstract}

Atomic forcemicroscopy (AFM) is a well-established field of research that offers multiple measurements including surface topology mapping, magnetic force measurements and electric conductance imaging with high-spatial resolution in various environments, like a ir, vacuum and/or liquids modalities [1-2]. Studies of nanomechanical properties of biological molecules, orga nella and living cells may be performed through local indentation by the AFM probe [3-4]. Measurements performed on optically tra nsparent objects often require fluorescence imaging and the acquired AFM signal needs to be spatially correlated with the optical image [5].

In this work, we demonstrate a simple way of co ating AFM tips and cantilevers with fluorescent nanodiamonds (NDs), which enables their localization using fluorescence microscopy techniques. Standard silicon AFM probes were dip-coated in a suspension of NDs in isopropyl alcohol and the NDs used contained two types of color centers: nitrogen-vacancy (NV) and nitrogenvaca ncy-nitrogen (NVN, also designated a s H3). They can be easily imaged under blue (for NVN centers) or green (for NV centers) light excitation with their fluorescence detected in the green and red wavelength bands, respectively. In the case of $\mathrm{NV}$ diamonds, additional measurement modalities are possible, e.g., magnetic field sensing [6]. While there have been many examples of scanning probe microscopy with dia mond probes, including the use of single NV centers for nanoscale magnetometry [7-15], we focus here on the customization of more standard, silicon-based probes.

NV color centers are atom-like systems finding m any practical applications in science and na notechnology owing to their bright and stable fluorescence and spin properties, particularly when they are in their negative electronic charge state, $\mathrm{NV}^{-}$[16-18]. The $\mathrm{NV}^{-}$defect possesses a spin-triplet ground state which enables magnetic and electric field sensing using so-called, optically detected magnetic resonance (ODMR) [19], [20]. Green laser light is used for the spin polarization and

http://www.photonics.pl/PLP readout while a microwave (MW) field a round $2.87 \mathrm{GHz}$ is used for the spin manipulation. Small $(\sim 1-10 \%)$ fluorescencedips are observed when the MW field is in resonance with the NV spin transition. External fields shift the resonance frequency which is monitore d by the fluorescence change. The ODMR spectrum can be used to determine the magnitude and, in some a rrangements, even the vector orientation of the perturbing field.

The spatial resolution of a magnetometer based on ND with an ensemble of NV centers, is a function of the size of the crystalused and its distance from a field source. Thus, to achieve good spatial resolution, placing NDs close to the tip apex is desired.

Our experiments were performed with commercially available silicon AFM probes (type PPP-CONTR, Na nosen sors). The overall chip size is $3.4 \times 1.4 \times 0.3 \mathrm{~mm}$ with the cantilever thickness, length, and width being 2.0 $\pm 0.1 \mu \mathrm{m}, 450 \pm 10 \mu \mathrm{m}$, and $50 \pm 7.5 \mu \mathrm{m}$, respectively. The tip height is within $10-15 \mu \mathrm{m}$ range. For dipcoating, the chip tip was carefully immersed for a few seconds into a suspension of nano-diamonds (MDNVN1 $\mu \mathrm{m}$ powder with a $750 \mathrm{~nm}$ mean particle size, Ada mas Na notechnologies Inc.) in isopropyl a lcohol and then allowed to dry in air. This type of ND powder contains on average $\sim 2$ ppm of NVN centers and estimated $\sim 0.1 \mathrm{ppm}$ of $\mathrm{NV}$ centers. Importantly, observations under the microscope indicated fluorescence of individual crystals being dominated by eit her the NV or NVN centers. Therefore, to achieve a satisfactory coating with less abundant NV diamonds, the dipping process was repeated, and two repetitions were found to be typically sufficient.

Fluorescence imaging of coated ca ntilevers and ODMR measurements have been performed with a wide-field microscope setup schematically presented in Fig. 1. Excitation of theNV color centers is ensured by a green (532 nm), PM-fiber-coupled la ser (532-L-OE-115-1 W, Integrated Optics) with an output power of $60 \mathrm{~mW}$. The laser beam after the fiber was expanded to $\sim 9 \mathrm{~mm}$ diameter and collimated, and then focused at the back focal plane of the microscope objective (LUCPLFLN $40 \mathrm{x}, \mathrm{NA}=0.6$, Olympus) with a help of a dichroic mirror (DMLP567, Thorlabs). Fluorescence light collected by the objective was filtered by a 600-nm long-pass filter (FEL0600, Thorlabs) and projected onto a CMOS camera 


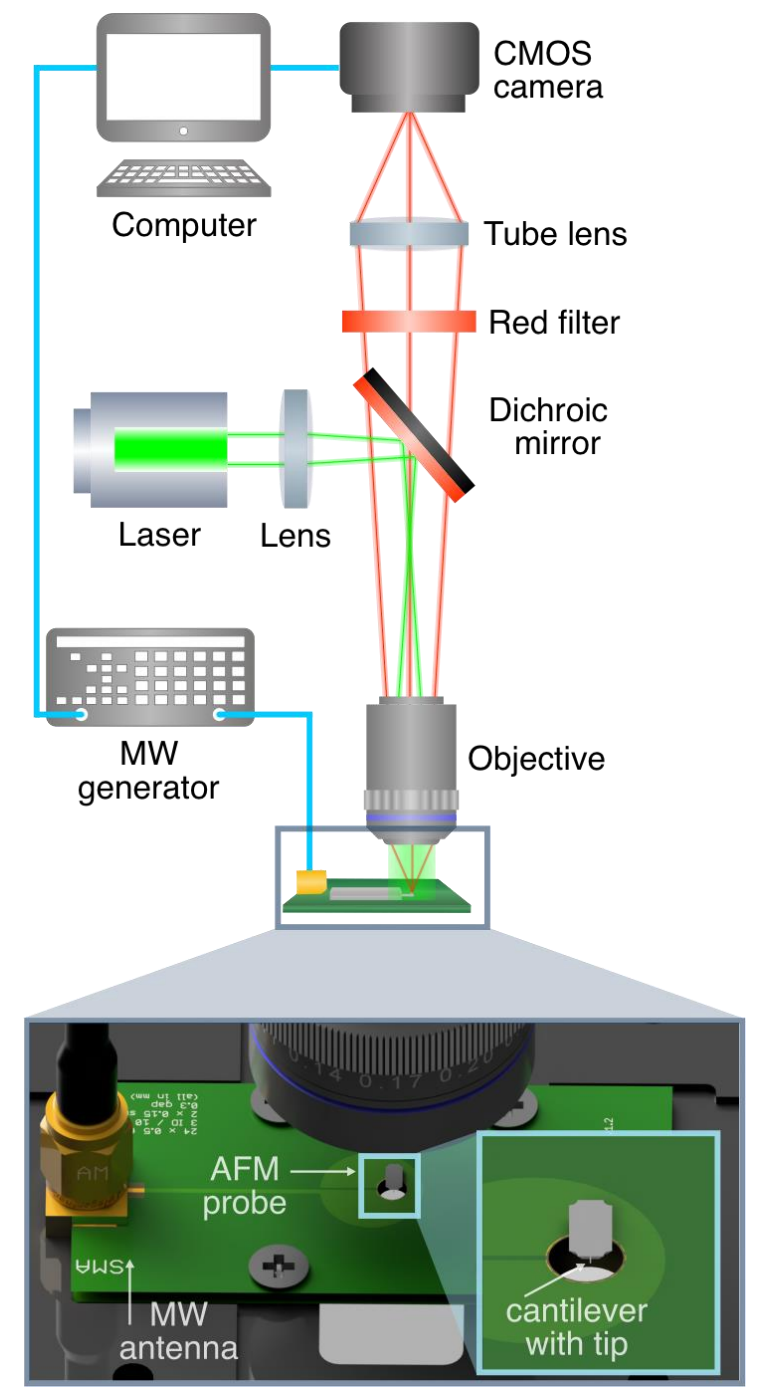

Fig. 1. Experimental apparatus for wide-field fluorescence microscopy and ODMR measurements. Inset shows the position of the AFM chip on the MW antenna.

sensor (IDS, UI-3240CP Rev. 2) using a 100-mm focal distance lens, which resulted in a field of vie w of $305 \times$ $244 \mu \mathrm{m}$. For the ODMR measurements, the MW field was generated using a signal generator (SG396, Stanford Resea rch Systems), amplified by a high-power amplifier (ZHL-16W-43+, Mini-Circuits) and delivered to a lo opgap type microstrip patch antenna [21] which has a 2mm-diameter central hole size and ensures spatially uniform magnetic field over the whole cantilever a rea (see the inset in Fig. 1). An additional coil (not shown in Fig. 1) was used for a pplying a static magnetic field of up to $1 \mathrm{mT}$ induction.

The fluorescence image of the ND-coated AFM cantilever is shown in Fig. 2. Under green light illumination, NDs containing NV centers are well visible as bright spots located randomly on the surface of the cantilever, which enables a convenient way of localizing the structure with a florescencemicroscope. Additionally,

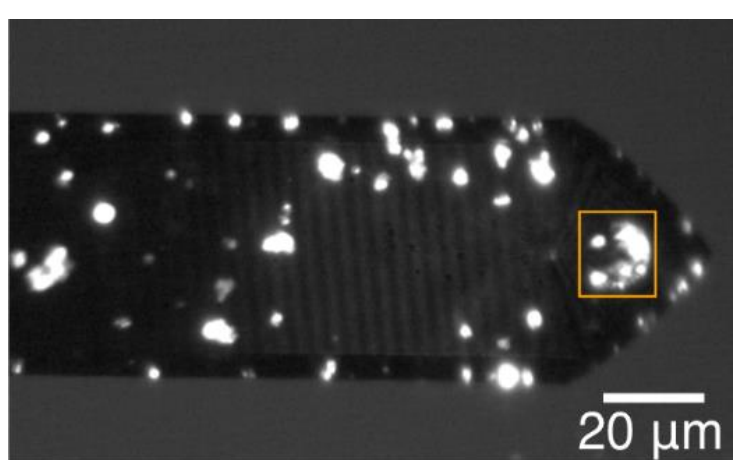

Fig. 2. Fluorescence image of the AFM cantilever coated with $750-\mathrm{nm}$-size nanodiamonds. The orange square marks the area from where the ODMR signal was collected.

there is a number of NDs visible a round the tip a rea marked with the orange square in Fig. 2. The tip ex tend s there from the cantilever plane towards the microscope objective and, due to the limited field depth of the optical setup, some NDs do not form a sharpim age. Howe ver, this doesn't preclude ODMR mea surements as long as fluorescence is integrated over the area containing a blurred image of all NDs. If the integration a rea is set too small, any drifts occurring during the MW frequency scans may cause fluorescence signal variations which can ea sily hinder the visibility of theODMR signal.

Figure 3 shows the ODMR signa ls a cquired for sev eral magnetic field values with the fluorescence signal integrated over the region marked in Fig. 2. While in a zero ma gnetic field the measured nanodiamond ODMR spectrum resembles closely the single-crystalline one [16], the spectra recorded in non-zero fields are significantly different. Rather than exhibiting discrete pairs of resonances corresponding to four possible NV orienta tions a long the [111] crystallogra phic direction in a bulk diamond, they are inhomogeneously broadened because of the random orientation of fluorescing NDs with respect to the direction of the magnetic field. Beca use of a dding fluorescence from multiple ra ndomly oriented NDs, the orientation of the magnetic field vector cannot be retrieved. However, the information on the field ma gnitude still can be extracted from the overall width of the spectrum [22], [23]. For the accurate re ad out of the field strength, this requires multiple, truly randomly oriented NDs contributing to the signal, but a less rigorous or qualitative magnetic field mapping is fea sible with any number of NDs.

In this work, we have demonstrated a simple and efficient method of making the AFM chips visible under a fluorescence microscope by dip-coating them with fluorescent NDs. The method can be useful for the localization of the AFM tip position with respect to optically transparent but fluorescing samples. More over, we have successfully recorded ODMR spectra from a group of NDs located around the pyramidal tip of the 


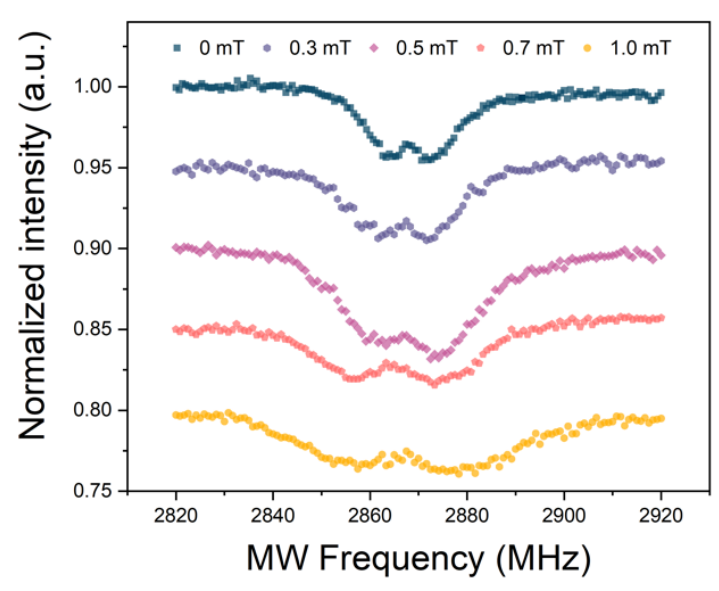

Fig. 3. ODMR signal for different value of magnetic fields. The visible broadening of a signal is caused by an increase in the applied magnetic field.

cantilever. This enables performing local magnetic field and temperature measurements with the same chip used for AFM mea surements. The spatial resolution in the case of ND magnetometry is strongly dependent on the size of NDs and their positioning around the tip and may be improved by applying smaller diamond particles and limiting the fluorescencecollection area to the tip a pex. Since the NDs a re addressed optically, the ultimate lim it on spatial resolution is coming from the optical diffraction limit and is on the order of hundreds of na nometers. Despite offering significantly worse spatial resolution than the AFM, this modality may find practical applications for discerning nanostructures characterized by similar topography but different magnetic properties.

Authors acknowledge stimulating discussions with Robert Bogdanowicz and Marta Targosz-Korecka. This work was supported by the Polish National Science Centre under Grants No.2016/21/B/ST7/01430 and the Foundation for Polish Science under the project TeamNET No. POIR.04.04.00-00-1644/18.

\section{References}

[1] G. Binnig, C. F. Quate, C. Gerber, Phys. Rev. Lett. 56, 930 (1986).

[2] F.J. Giessibl, Rev. Mod. Phys. 75, 949 (2003).

[3] S. Kasas, G. Dietler, Eur. J. Appl. Physiol. 456, 13 (2008).

[4] C. Roduit et al., Biophys. J. 97, 674(2009).

[5] L.A. Kolodny et al., Anal. Chem. 73, 1959(2001).

[6] L. Rondin et al., Rep. Prog. Phys. 77, 056503 (2014).

[7] C.L. Degen, Appl. Phys. Lett. 92, 243111 (2008).

[8] J.M. Taylor et al., Nat. Phys. 4, 810 (2008).

[9] J.R. Maze et al., Nature 455, 644 (2008).

[10] L. Rondin et al., Appl. Phys. Lett. 100, 153118 (2012).

[11] J.P. Tetienne et al., Science 344, 1366(2014).

[12] R. Nelz et al., Appl. Phys. Lett. 109, 193105(2016).

[13] G. Balasubramanian et al., Nature 455, 648(2008).

[14] P. Maletinsky et al., Nat. nanotechnol. 7,320 (2012).

[15] L. Thiel et al., Nat. nanotechnol. 11, 677 (2016).

[16] F. Jelezko et al., Appl. Phys. Lett. 81, 2160(2002).

[17] M. W. Doherty et al., Phys. Rep. 528, 1 (2013).

[18] C. Kurtsiefer, S. Mayer, P. Zarda, H. Weinfurter, Phys. Rev. Lett. 85, 290 (2000).

[19] A. Gruber, A. Dräbenstedt, C. Tietz, L. Fleury, J. Wrachtrup, C. Von Borczyskowski, Science 276, 2012 (1997).

[20] F. Dolde et al., Nat. Phys. 7, 459 (2011).

[21] K. Sasaki et al., Rev. Sci. Instrum. 87, 053904 (2016).

[22] A.M. Wojciechowski et al., Materials 12,2951(2019).

[23] I.V. Fedotov et al., Opt. Lett. 39, 6755(2014). 